\title{
Long-term Protection Against the Hepatitis B Virus Detected Through an Early Response to a Booster Dose Injection
}

\author{
Hiva Saffar ${ }^{1}$, Ali-Reza Khalilian ${ }^{2}$, Mohammed-Jafar Saffar ${ }^{3}$ And AbolghaSem AJami ${ }^{4}$ \\ From the Department of ${ }^{1}$ Pathology, Shariati Hospital, Tehran University of Medical Sciences, Tehran; and Departments of \\ ${ }^{2}$ Biostatistics, ${ }^{3}$ Pediatric Infectious Diseases Ward and Antimicrobial Resistance Nosocomial Infection Research Center, and \\ ${ }^{4}$ Immunology and Molecular and Cellular Biology Research Center, Mazandaran University of Medical Sciences, Sari; Iran.
}

Correspondence to: Dr Mohammed-Jafar Saffar, Pediatric Infectious Diseases Ward and Antimicrobial Resistance Nosocomial Infection Research Center, Mazandaran University of Medical Sciences, Sari, Iran.

Saffar@softhome.net.

Received: January 12, 2016;

Initial review: March 26, 2016;

Accepted: September 13, 2017.
Objective: To determine the duration of protection conferred by the hepatitis B (HB) vaccination and the necessity of a booster dose. Methods: Immediately after the initial blood sampling, 252 youths (aged 18.8-20.5 years, $52 \%$ females) with a history of neonatal HB vaccination with one dose of the $H B$ vaccine received a booster. Serum concentrations of antibodies against the HB surface antigen were assessed in samples collected before and 10-14 days after the booster. Seroconversion from concentrations $<10$ to $\geq 10 \mathrm{IU} / \mathrm{L}$ were defined as a positive immune response. Results: Of the 252 participants, 131 were serosusceptible and 114 responded. Conclusions: Nearly $90 \%$ of young people preserved their long-term protection; the results of this study do not support the use of an HB booster vaccination.

Keywords: Booster vaccination, HBV vaccine, Immune memory, Long-term protection.
$\mathrm{H}$ epatitis B immunization beginning in infancy is reported to protect at least 20 years even without a booster dose [1-4], but few other studies have suggested that vaccinations given to infants might not be sufficient to offer long-lasting protection [5-7]. Therefore, it is reasonable to determine whether vaccinated infants maintain their protection against $\mathrm{HB}$ until they reach an age where their risk of HB infection increases due to their lifestyles and professional exposure or if they need a booster vaccination to sustain their immunity into adulthood. Protection against HB infection is mediated through the specific antibodies against HB surface antigen (anti-HBs) [8]; therefore, an indirect method to investigate the presence of immune protection and memory is to look for an anamnestic response following the administration of a booster dose to the vaccinees that had lost their acquired humoral immunity (antibodies) over time. A rapid and prominent increase in anti-HBs concentrations a short time after boosting indicates the presence of specific immune memory and likely protection against HB infection [9-11]. In Iran, universal neonatal $\mathrm{HB}$ immunization was incorporated in the National Program of Immunization since March 1993, scheduled at birth, 1.5 months and 9 months of age. This study was designed to evaluate the $\mathrm{HB}$ infection seromarkers among young adults, and also to investigate their immune response to a booster dose injection.

\section{METHODS}

Study participants were volunteers selected from a previous sero-epidemiological study in young adults who had been vaccinated 20 years earlier [12]. In that study, 510 youths with a documented history of complete HB vaccination starting at birth "as the first cohort of Iranian neonates involved in the national program" were the participants. In the present study, we randomly selected 252 people out of 510 cases of the former study. The study was approved by the Ethics Committees of the Mazandaran and Tehran Universities of Medical Sciences. Written informed consent was obtained prior to the study.

Immediately after the first blood sampling, all participants received a dose of the HB vaccine. Ten to 14 days later, second blood samples were obtained from each participant. Pre- and post-booster sera were analyzed for their concentrations of the HB surface antigen ( $\mathrm{HBsAg}$ ), and antibodies against the HBsAg (anti-HBs) and the HB core antigen (anti-HBc), using a quantitative enzyme-linked immunosorbent assay method with commercial kits. Concentrations were expressed as $1 \mathrm{U} / \mathrm{L}$, and titers $>370 \mathrm{IU} / \mathrm{L}$ were not further discriminated. The participants were divided into four groups based upon their anti-HBs levels in the prebooster sera as follows: G1: undetectable titer $(<2 \mathrm{IU} / \mathrm{L})$; 
G2: non-protected titer (2-9.9 IU/L); G3: low protection, (10-99 IU/L); and G4: highly protected, titers ( $\geq 100 \mathrm{IU} /$ L). Anti-HBs concentrations ( $\geq 10 \mathrm{IU} / \mathrm{L})$ were considered protective, and concentrations $(\geq 300 \mathrm{IU} / \mathrm{L})$ without a positive anti-HBc were arbitrarily defined as natural boosting. Participants with positive anti-HBc antibodies with/without positive anti-HBs were considered to have a subclinical breakthrough infection. A positive immune response was defined as seroconversion from titers $<10$ to $\geq 10 \mathrm{IU} / \mathrm{L}$, while an anamnestic response included the acquisition of an anti-HBs concentration $\geq 100 \mathrm{IU} / \mathrm{L}$ in those subjects with titers $<10 \mathrm{IU} / \mathrm{L}$ or a $\geq 4$-fold increase in titers in participants with anti-HBs levels 10-99 IU/L before boosting. The mean concentration of antibodies (MCA) and the proportion of responders to boosting in each group were also calculated. Chi-square and independent t-tests were used to compare the proportion of responders and the MCA produced after boosting in the different groups. A $P$ value $<0.05$ was considered statistically significant.

\section{Results}

A total of 252 young adults with an age range from 18.820.5 years (52\% females) were enrolled in this study. After excluding 9 participants with anti-HBc positivity, the relative proportion of allocated subjects in each group was as follows: G1, 88; G2, 43; G3, 41; and G4, 71 individuals. In the G4 participants, $41(16.2 \%)$ showed antibody concentrations $>300 \mathrm{IU} / \mathrm{L}$. Ten to 14 days after the booster injection, 114 of $131(87 \%)$ of the nonprotected individuals mounted a positive immune response and were seroconverted. Participants whose pre-booster anti-HBs levels were from 2-9.9 IU/L had a higher probability of showing a response to boosting in comparison to participants levels $<2 \mathrm{IU} / \mathrm{L}$. Of the 41 participants in the G3 group, $39(95.1 \%)$ showed an anamnestic response, and their post-booster MCA levels increased significantly $(P=0.001)$. None of the G4 group responded to the booster. Finally, $90 \%$ of the participants retained their vaccine protection for 20 years.

\section{Discussion}

To better determine the kinetics of vaccine-induced antiHBs antibody titer and the prevalence of boostability among vaccinated subjects over time, a comparison was made between this study's findings (a preservation rate of $>44 \%$ seroprotection and an $87 \%$ response rate to boosting) and the results of our earlier study, which was performed 10 years ago in another region of the province [13] in the same cohort of Iranian infants vaccinated with the same vaccine and schedule. The results indicated that the prevalence of seroprotection rates decreased significantly but the boostability of the vaccinees did not change significantly. In this study, there was a direct correlation between pre-booster anti-HBs titers and the magnitude of the immune response to the booster injection; a lower response was seen in those with undetectable titers. Similar findings were earlier reported [14]. These findings may suggest that higher numbers of memory cells may be impaired or even disappeared in these vaccinated subjects.

The results of several long-term follow-up studies which investigated the persistence of specific antibodies and the presence of immune memory in persons vaccinated with different schedules and vaccines living in different settings of HB infection have been published [17]. While most studies have reported that nearly half of the vaccinees preserved their seroprotection 15-23 years after vaccination and the majority successfully responded to a booster vaccination (even those without detectable antibody titers) [1-4], some other studies have shown a very short duration of antibody persistence and poor immune memory [5-7]. Studies conducted in low-risk settings with comparable follow-up periods have generally shown lower rates of seroprotection and anamnestic responses than those in high-endemic countries. In a 15-year prospective study by Hammitt, et al. [5] only $5 \%$ of the vaccinated subjects preserved their seroprotection and an anamnestic response was observed in $51 \%$ of the boosted participants. These rates were $24 \%$

TABLE I Pattern Of IMMUne Response to A Booster Dose 20 Years After Primary Hepatitis B Immunization

\begin{tabular}{llclr}
\hline Anti-HBs antibody concentration (IU/L) & & No. of subjects & MCA*(SD) IU/L & $\begin{array}{c}\text { Number } \\
\text { of responders (\%) }\end{array}$ \\
\cline { 3 - 4 } $\mathrm{G}_{1}:$ concentration $<2$ & 88 & $0.6(0.44)$ & $193.6(157.8)$ & $74(84.2)$ \\
$\mathrm{G}_{2}:$ concentration 2-9.9 & 43 & $4.9(2.05)$ & $309.7(119.2)$ & $40(93.1)$ \\
$\mathrm{G}_{3}:$ concentration 10-99 & 41 & $35.6(25.1)$ & $342.58(96.6)$ & $39(95.1)$ \\
$\mathrm{G}_{4}:$ concentration $\geq 100$ & 71 & $326.67(82.60)$ & $355.06(33.07)$ & Not applicable \\
\hline
\end{tabular}

*MCA: Mean concentration of antibodies; P value: G1 vs. G2: 0.07 for the proportion of responders and $<0.001$ for MCA. 


\section{WhAT THIS STUdY AdDS?}

- Primary vaccination with hepatitis B vaccine conferred adequate protection against disease at least for 20 years, and booster dose is not required even in the low endemicity area.

and $92 \%$, respectively, in a study conducted in children in the United States by Middleman, et al. [4].

In contrast to most long-term prospective studies, in our cross-sectional study, the primary immune response and the initial anti-HBs antibody concentration achieved after priming were not studied. Therefore, our estimation for the natural boosting prevalence contained an arbitrary cut-off that may result in the under- or over-estimation of the natural boosting rate.

In conclusion, our study results indicate that infant HB vaccination provides long-term protection up through 20 years of age in the majority of vaccinated infants. These findings are in accordance with the current recommendation by most experts that a universal routine booster vaccination in the general population is not required for up to 20 years. However, the screening of vaccinated young adults at increased risk of exposure to $\mathrm{HB}$ infection to evaluate their immunity status and possible requirement for booster vaccination may still be required $[9-11,15]$.

Acknowledgement: K. Shams-Esfandabad, J. Shojaei, and the health staff in the health centers affiliated with the Deputy of Health (Mazandaran University of Medical Sciences) for assistance in data collection and blood sampling; and $\mathrm{M}$. Sotudeh-Anvari for her help with the laboratory testing and data collection.

Contributors: HS and MJS: conceptualization, design, data collection, and writing the paper; AA: design and laboratory testing; A-RK: statistical analysis.

Funding: Mazandaran and Tehran Universities of Medical Sciences; Competing interest: None stated.

\section{REFERENCES}

1. Poovorawan Y, Chongsrisawat V, Theamboonlers A, Leroux-Roels G, Kuriyakose S, Leyssen M, et al. Evidence of protection against clinical and chronic hepatitis $\mathrm{B}$ infection 20 years after infant vaccination in a high endemicity region. J Viral Hepat. 2011;18:369-75.

2. McMahon BJ, Dentinger CM, Bruden D, Zanis C, Peters $\mathrm{H}$, Hurlburt D, et al. Antibody levels and protection after hepatitis B vaccine: Results of a 22-year follow-up study and response to a booster dose. $\mathrm{J}$ Infect Dis. 2009;200:1390-6.

3. Wu Q, Zhuang GH, Wang XL, Wang LR, Li N, Zhang M.
Antibody levels and immune memory 23 years after primary plasma-derived hepatitis B vaccination: results of a randomized placebo-controlled trial cohort from China where endemicity is high. Vaccine. 2011;29:2302-7.

4. Middleman AB, Baker CJ, Kozinetz CA, Kamili S, Nguyen $\mathrm{C}, \mathrm{Hu} \mathrm{DJ}$, et al. Duration of protection after infant hepatitis B vaccination series. Pediatrics. 2014;133:e1500-7.

5. Hammitt LL, Hennessy TW, Fiore AE, Zanis C, Hummel $\mathrm{KB}$, Dunaway E, et al. Hepatitis B immunity in children vaccinated with recombinant hepatitis $B$ vaccine beginning at birth: A follow-up study at 15 years. Vaccine. 2007;25:6958-64.

6. Chan PK, Ngai KL, Lao TT, Wong MC, Cheung T, Yeung AC, et al. Response to booster doses of hepatitis B vaccine among young adults who had received neonatal vaccination. PLoS One. 2014;9:e107163.

7. Jan CF, Huang KC, Chien YC, Greydanus DE, Davies HD, Chiu TY, et al. Determination of immune memory to hepatitis B vaccination through early booster response in college students. Hepatology. 2010;51:1547-54.

8. Pichichero ME. Booster vaccinations: Can immunologic memory outpace disease pathogenesis? Pediatrics. 2009;124:1633-41.

9. Leuridan E, Van Damme P. Hepatitis B and the need for a booster dose. Clin Infect Dis. 2011;53:68-75.

10. FitzSimons D, Hendrickx G, Vorsters A, Van Damme P. Hepatitis B vaccination: A completed schedule enough to control HBV lifelong? Vaccine. 2013;31:584-90.

11. World Health Organization.Hepatitis B Vaccine.WHO position paper.Wkly Epidemiol Rec. 2009;48:405-20.

12. Saffar H, Ajami A, Saffar MJ, Shojaei J, Sotudeh-Anvari M, Shams-Esfandabad K, et al. Prevalence of hepatitis B virus seromarkers in young adults vaccinated at birth: Impact on the epidemiology of hepatitis B infection in iran. Hepat Mon. 2014;14:e17263.

13. Saffar MJ1, Rezai MS. Long-term antibody response and immunologic memory in children immunized with hepatitis B vaccine at birth. Indian Pediatr. 2004;41:1232-7.

14. Spradling PR, Xing J, Williams R, Masunu-Faleafaga $Y$, Dulski T, Mahamud A, et al. Immunity to hepatitis B virus (HBV) infection two decades after implementation of universal infant $\mathrm{HBV}$ vaccination: Association of detectable residual antibodies and response to a single HBV challenge dose. Clin Vaccine Immunol. 2013;20:559-61.

15. Poorolajal J, Mahmoodi M, Majdzadeh R, NasseriMoghaddam S, Haghdoost A, Fotouhi A. Long-term protection provided by hepatitis B vaccine and need for booster dose: A meta-analysis. Vaccine. 2010;28:623-31. 